\title{
Characterization of the amino-terminal domain of Mx2/MxB-dependent interaction with the HIV-1 capsid
}

\section{Dear Editor,}

More than 50 years have passed since the myxovirus resistance $(M X)$ genes were first discovered and found to suppress infection with influenza viruses in mice (Lindenmann, 1962). Like most mammals, mice carry two $M X$ genes, $M X 1$ and $M X 2$, which have arisen by gene duplication; both of these genes exhibit antiviral activity against a wide range of viruses (Liu et al., 2013). Humans also have two $M X$ genes, encoding the $M \times A$ and $M \times B$ proteins, which are interferon-induced, dynaminlike large molecular weight guanosine triphosphatases (GTPases). The antiviral functions of MxA have been deeply explored: MxA can protect cells from infection by multiple groups of pathogenic DNA and RNA viruses, such as influenza $A$ virus and hepatitis $B$ virus (Liu et al., 2013). In contrast, Mx2, although closely related to $\mathrm{MxA}$ ( $63 \%$ amino acid [aa] sequence identity), appears to have lost its antiviral function and has been recognized as playing other cellular roles, since it does not suppress the viruses tested (Melen et al., 1996).

Recently, Mx2 has been shown to serve as an inhibitor of human immunodeficiency virus type-1 (HIV-1) (Goujon et al., 2013; Kane et al., 2013; Liu et al., 2013). Mx2 restricts HIV-1 infection at a relatively late post-entry phase (Goujon et al., 2013; Kane et al., 2013) and leads to a reduced level of integrated viral DNA (Liu et al., 2013). The N-terminal 91-aa domain of Mx2 has been identified as a critical determinant of Mx2's antiviral activity (Busnadiego et al., 2014). Interestingly, several mutations in the HIV-1 viral capsid (CA) region of Gag can overcome Mx2-mediated suppression (Goujon et al., 2013; Kane et al., 2013; Liu et al., 2013). Thus, Mx2 may bind to the HIV-1 core and inhibit the early events in HIV-1 binding, thereby restricting viral infection. However, there is still no evidence showing that Mx2 directly binds to the HIV-1 capsid. Whether capsid binding of Mx2 requires cellular co-factors and/or higher-order assemblies of $\mathrm{CA}$ is also unknown. In this study, we have obtained a stable $\mathrm{Mx} 2$ protein containing the $\mathrm{N}$-terminal 91-aa domain. Furthermore, we have observed that purified $M \times 2$ recombinant proteins bind directly to HIV-1 CA assemblies in vitro. The N-terminal 83-aa domain of $M \times 2$ is apparently critical for this interaction.

Human MxA and Mx2 share a similar aa sequence and domain architecture (Liu et al., 2013; Melen et al., 1996). The crystal structure of MxA indicates that it includes a $G$ domain that binds and hydrolyzes GTP; a hinge-like "bundle signaling element (BSE)" that connects the G domain to the elongated stalk domain; and the stalk domain, which is involved in self-assembly and oligomerization (Gao et al., 2010; Gao et al., 2011) (Fig. 1A). A unique feature of $M \times 2$ is that it exhibits a longer N-terminal domain, including an NLS (N-terminal 25 aa).

To identify the contribution of the diverse Mx2 domains to capsid binding, we generated and screened a series of deletion constructs of Mx2 with an N-terminal His-Sumo-tag (Fig. S1A) and characterized the expression, solubility, stability, and oligomerization behavior of these constructs (Table S1). We found that full-length $M \times 2$ was difficult to obtain in $E$. coli. The expression levels of all the other deletion constructs (1-387, 1-413) were greatly reduced, but $\Delta 1-83$ exhibited a highly improved expression level (Fig. S1B), indicating that the $\mathrm{N}$-terminal 83-aa domain of Mx2 may cause low expression and instability in solution (Table S1). Other deletion constructs (84-387, 84-413) without the $\mathrm{N}$-terminal 83-aa domain showed high expression levels (Fig. S1B), but it exhibited poor solubility and stability (Fig. $\mathrm{S} 1 \mathrm{C}$ ), indicating that the completeness of BSE plays a significant role in maintaining the structure of the $M \times 2$ constructs. Thus, we were unable to obtain stable constructs with the $\mathrm{N}$-terminal 83 -aa domain.

To overcome this problem, on the basis of previous reports (Chappie etal., 2010) and the structure of MxA (Gao et al., 2011), we engineered a minimal GTPase-BSE fusion protein (GF) that connected residues 84-413 and residues 683-715 from human Mx2 via a flexible linker (Fig. S1A). GF eluted as a monodispersed peak from a size-exclusion column and had much better solubility $(>20 \mathrm{mg} / \mathrm{mL}$ ) than did residues $84-387$ and 84 413 (Fig. S1C). In the next phase, we added the N-terminal 83-aa domain of $\mathrm{M} \times 2$ to $\mathrm{GF}$ in order to generate an $\mathrm{N}$-terminalGTPase-BSE fusion protein (N-GF). His-Sumo-N-GF showed a highly improved expression level ( $>20 \mathrm{mg} / \mathrm{L}$ in $E$. coli) and solubility when compared to other deletion constructs with the $\mathrm{N}$-terminal 83-aa domain (Fig. S1C). After removing the Sumotag, we saw a reduction in the stability of the N-GF proteins, but $\mathrm{N}$-GF eluted as a monodispersed peak from a size-exclusion 


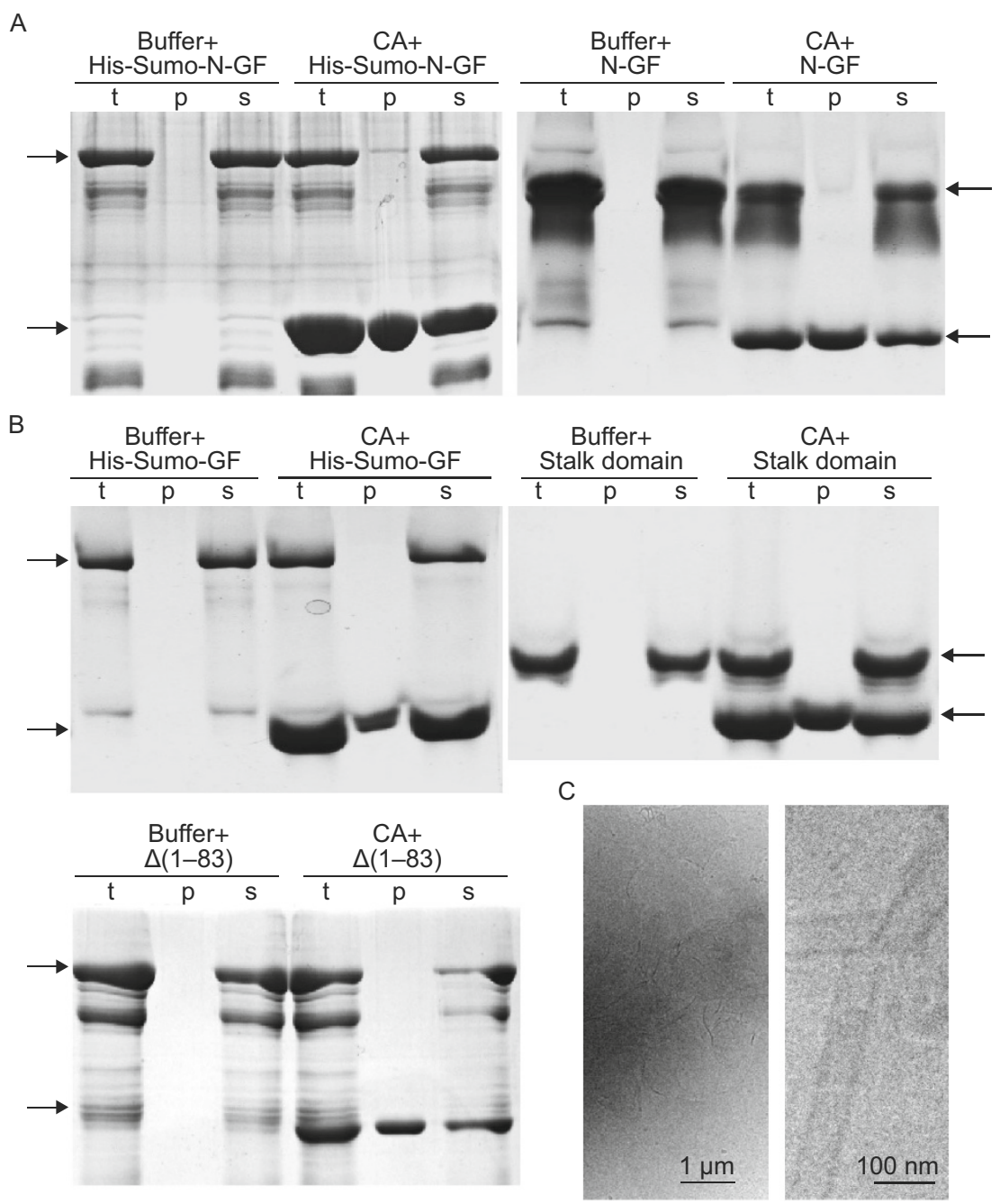

Figure 1. Interactions of Mx2 variants with CA tubular assemblies. (A) Binding of His-sumo-N-GF and N-GF to pre-assembled wild-type CA tubes. Binding reactions were analyzed by SDS-PAGE using CA tubular assemblies at $80 \mu \mathrm{mol} / \mathrm{L}$ or binding buffer alone, incubated with His-sumo-N-GF and N-GF $(79 \mu \mathrm{mol} / \mathrm{L})$. Samples of the reaction mixture before centrifugation (t), of the supernatant (s), and of the pellet ( $p$ ) are detected alone. (B) Binding of Mx2 constructs to preassembled wild-type CA tubes. Binding reactions were the same as in (A). No binding of any Mx2 construct without the N-terminal 83-aa domain was found. (C) Cryo-EM images of pre-assembled wild-type CA tubes used in the Mx2-CA binding experiments.

column (Table S1). Thus, we successfully generated a stable fusion construct of $\mathrm{M} \times 2$ for use in detecting further binding.

It had previously been demonstrated that assembled HIV1 CA-NC tubular complexes can interact with full-length MX2 and its truncations containing the $\mathrm{N}$-terminal 91-aa (isolated from HEK293T cell lysates) (Fricke et al., 2014). To further clarify whether Mx2 directly binds to HIV-1 CA assemblies (at $20 \mu \mathrm{mol} / \mathrm{L}$ ) in vitro, we tested purified His-Sumo-N-GF and $\mathrm{N}-\mathrm{GF}(79 \mu \mathrm{mol} / \mathrm{L})$ in precipitation assays with preassembled $\mathrm{CA}$ tubes. The results clearly indicated that the N-GF proteins co-pellet with assembled $C A$, revealing that the binding process does not require other cellular co-factors (Fig. 1A); thus, established that Mx2 directly binds to HIV-1 CA tubes in vitro.

We performed a further dissection of the interactions between the $M \times 2$ constructs and $C A$ to address the contribution of the different Mx2 domains to capsid binding. Purified GF, $\Delta 1-83$, and the stalk domain $(79 \mu \mathrm{mol} / \mathrm{L})$ were each tested in the precipitation assay with preassembled $C A$ tubes $(20 \mu \mathrm{mol} / \mathrm{L})$. Essentially no binding was observed for $\mathrm{GF}$, $\Delta 1-83$, or the stalk domain under the same assay conditions (Fig. 1B). All CA tubes were prepared as previously described (Yang et al., 20122012; Hung et al., 2013) and were confirmed by cryo-electron microscopy (cryo-EM) (Fig. 1C). The findings described above indicate that $M \times 2-C A$ binding requires the $\mathrm{N}$-terminal 83-aa of $\mathrm{M} \times 2$. The $\mathrm{N}-\mathrm{GF}$ does not contain the stalk domain, but it is apparently sufficient to bind HIV-1 CA assembly. On the other hand, the stalk domain alone showed no binding to the assembled CA tubes (Fig. 1B).

HIV-1 capsid (CA) proteins can assemble into closed fullerene cones or helical tubes in vivo and in vitro. It is widely known that tripartite motif protein isoform 5 alpha 


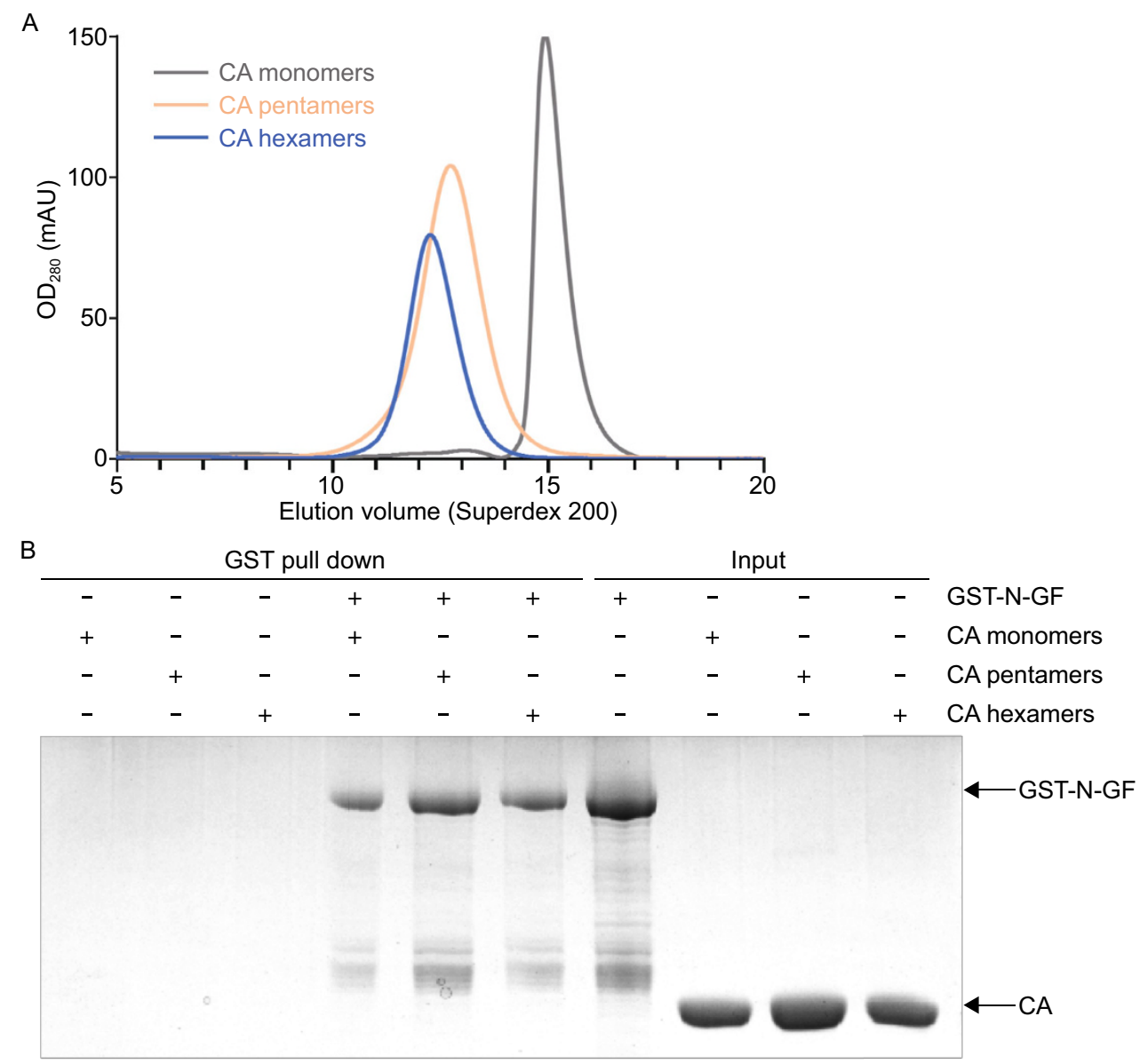

Figure 2. GST Pull-down assays of GST-N-GF with CA hexamers, pentamers, and monomers. (A) Size-exclusion chromatographic profiles of individual CA hexamers, pentamers, and monomers. (B) Pull-down assays of GST-N-GF with CA hexamers, pentamers, and monomers. CA hexamers, pentamers, and monomers, incubated with GST-N-GF-bound glutathionesepharose beads, showed no more interaction than those incubated with glutathione-sepharose beads alone.

(TRIM5 $\alpha$ ) is an antiviral protein that restricts infection of HIV-1 by binding to the viral capsid. The TRIM5 $\alpha-C A$ binding interaction requires an assembled capsid lattice, since individual CA monomers do not have an appreciable affinity for TRIM5a (Stremlau et al., 2006). It is the shape of the HIV-1 capsid that is recognized by the TRIM5a protein (Yang et al., 2012). Having confirmed the binding of Mx2-CA assemblies, we wanted to investigate whether, like TRIM5 $\alpha, M \times 2$ binds to the viral capsid by recognizing the shape of the higher-order assembled HIV-1 capsid lattice. Therefore, we over-expressed and purified individual hexamers (A14C/E45C/W184A/ $\mathrm{M} 185 \mathrm{~A})$, pentamers (A21C/E22C/W184A/M185A), and monomers of CA as previously described (Fig. 2A) (Pornillos et al., 2011), and separately examined the binding affinity between each of them and GST-N-GF by GST pull-down assays, detecting the results by SDS-PAGE. No complex formation was detected, even at very high concentrations of both proteins (Fig. 2B). This result demonstrates that $\mathrm{M} \times 2$ shows no detectable binding to CA hexamers, pentamers, or monomers, implying that a higher-order lattice of CA tubes is required for an efficient $\mathrm{Mx2}-\mathrm{CA}$ interaction.
In the current study, we have demonstrated for the first time that a direct interaction occurs between $\mathrm{Mx} 2$ and the $\mathrm{HIV}-1$ capsid. The N-terminal region of Mx2 is critical for this interaction. We identified a fusion protein of $\mathrm{Mx} 2$ containing the N-terminal 83-aa and the complete BSE (N-GF), which was fundamental for the detection of $\mathrm{Mx2}-\mathrm{CA}$ binding in vitro. The N-GF construct may also facilitate further biochemical and structural studies of Mx2. The co-pelleting of purified N-GF and CA tubes, as well as domain mapping studies, showed that $\mathrm{Mx} 2$ directly recognizes $\mathrm{CA}$ assembles through its N-terminal 83-aa domain. Based on the results of pulldown assays, Mx2-CA binding requires the higher-order assembled capsid lattice, just as TRIM5a-CA binding does.

While this work was in progress, it was reported that assembled HIV-1 CA-NC tubular complexes can co-pellet Mx2 from whole-cell lysates (Fricke et al., 2014). However, there is no evidence to show that Mx2 directly binds to CA in vitro (i.e., whether capsid binding of $\mathrm{Mx} 2$ requires co-factors in cells). The contribution of the NC domain to the binding of Mx2 to higherorder assemblies of CA-NC was also not clear. Our data argue that there is direct interaction between $\mathrm{Mx} 2$ and HIV-1 CA. 
Interestingly, several mutations in the HIV-1 viral capsid (CA) region of Gag can overcome MX2-mediated suppression (Fricke et al., 2014; Liu et al., 2013). The CA-NC tubular complexes that are formed with mutations in CA that help HIV-1 escape from $M x 2$ restriction exhibit weakened interactions with Mx2 (Fricke et al., 2014). Thus, Mx2 may bind to the HIV-1 core and inhibit the process of HIV-1 infection.

It has been reported that $M \times 2$ variants $\Delta 572-715$ and $\Delta 623-715$ have lost their ability to oligomerize, and they fail to bind to CA-NC assemblies in vitro (Fricke et al., 2014). Our results show that although N-GF lacks the stalk domain involved in oligomerization, it still has the ability to bind $\mathrm{CA}$ tubes. This binding may occur because N-GF has the complete BSE, which is of great importance in maintaining normal Mx2 structure. These results suggest that although it is possible that the oligomerization of $\mathrm{Mx} 2$ produces a higher binding affinity for the viral capsid, oligomerization is not necessary for $\mathrm{Mx2}-\mathrm{CA}$ binding. Future studies will be required to explore the oligomerization functions that are required for $\mathrm{Mx2}$-mediated HIV-1 restriction (Haller et al., 2010).

\section{ACKNOWLEDGEMENT}

This work was supported in part by funding from the Chinese Ministry of Science and Technology (No. 2012CB911100 and No. 2013ZX0001-005) and Tianjin Research Program of Application Foundation and Advanced Technology (No. 14JCQNJC09400).

Jia Kong, Bo Xu, Wei Wei, Xin Wang, Wei Xie, and Xiao-Fang Yu declare that they have no conflict of interest. This article does not contain any studies with human or animal subjects performed by any of the authors.

Jia Kong ${ }^{1,2}$, Bo $\mathrm{Xu}^{1,2}$, Wei Wei $\mathrm{i}^{2,3,4}$, Xin Wang ${ }^{1,2}$, Wei Xie ${ }^{1,2 \bowtie}$, Xiao-Fang Yu ${ }^{1,2,3,4 \bowtie}$

${ }^{1}$ School of Life Science, Tianjin University, Tianjin 300072, China

${ }^{2}$ Collaborative Innovation Center of Chemical Science and Engineering, Tianjin 300072, China

3 Department of Molecular Microbiology and Immunology, Johns Hopkins Bloomberg School of Public Health, Baltimore, MD 21205, USA

${ }^{4}$ Institute of Virology and AIDS Research, First Hospital of Jilin University, Changchun 130061, China

$\square$ Correspondence: xiewei.xray@gmail.com (W. Xie), xfyu@tju. edu.cn (X.-F. Yu)

\section{OPEN ACCESS}

This article is distributed under the terms of the Creative Commons Attribution License which permits any use, distribution, and

Jia Kong and Bo Xu have contributed equally to this work.

Electronic supplementary material The online version of this article (doi:10.1007/s13238-014-0113-5) contains supplementary material, which is available to authorized users. reproduction in any medium, provided the original author(s) and the source are credited.

\section{REFERENCES}

Busnadiego I, Kane M, Rihn SJ, Preugschas HF, Hughes J, BlancoMelo D, Strouvelle VP, Zang TM, Willett BJ, Boutell C et al (2014) Host and Viral Determinants of Mx2 Antiretroviral Activity. J Virol 88:7738-7752

Chappie JS, Acharya S, Leonard M, Schmid SL, Dyda F (2010) G domain dimerization controls dynamin's assembly-stimulated GTPase activity. Nature 465:435-440

Fricke T, White T, Schulte B, de Souza Aranha Vieira D, Dharan A, Campbell E, Brandariz-Nunez A, Diaz-Griffero F (2014) Mx2 binds to the HIV-1 core and prevents the uncoating process of HIV-1. Retrovirology 11:68

Gao S, von der Malsburg A, Paeschke S, Behlke J, Haller O, Kochs G, Daumke O (2010) Structural basis of oligomerization in the stalk region of dynamin-like MxA. Nature 465:502-506

Gao S, von der Malsburg A, Dick A, Faelber K, Schröder, Gunnar F, Haller O, Kochs G, Daumke O (2011) Structure of myxovirus resistance protein $A$ reveals intra- and intermolecular domain interactions required for the antiviral function. Immunity 35:514-525

Goujon C, Moncorge O, Bauby H, Doyle T, Ward C, Schaller T, Hue S, Barclay W, Schulz R, Malim M (2013) Human MX2 is an interferon-induced post-entry inhibitor of HIV-1 infection. Nature 502:559-562

Haller O, Gao S, von der Malsburg A, Daumke O, Kochs G (2010) Dynamin-like MXA GTPase: structural insights into oligomerization and implications for antiviral activity. J Biol Chem 285:28419-28424

Hung M, Niedziela-Majka A, Jin D, Wong M, Leavitt S, Brendza KM, Liu X, Sakowicz R (2013) Large-scale functional purification of recombinant HIV-1 capsid. PLoS One 8:e58035

Kane M, Yadav S, Bitzegeio J, Kutluay S, Zang T, Wilson S, Schoggins J, Rice C, Yamashita M, Hatziioannou T et al (2013) MX2 is an interferon-induced inhibitor of HIV-1 infection. Nature 502:563-566

Lindenmann J (1962) Resistance of mice to mouse-adapted influenza A virus. Virology 16:203-204

Liu Z, Pan Q, Ding S, Qian J, Xu F, Zhou J, Cen S, Guo F, Liang C (2013) The interferon-inducible Mx2 protein inhibits HIV-1 infection. Cell Host Microbe 14:398-410

Melen K, Keskinen P, Ronni T, Sareneva T, Lounatmaa K, Julkunen I (1996) Human Mx2 protein, an interferon-alpha-inducible GTPase, contains a nuclear targeting signal and is localized in the heterochromatin region beneath the nuclear envelope. J Biol Chem 271:23478-23486

Pornillos O, Ganser-Pornillos BK, Yeager M (2011) Atomic-level modelling of the HIV capsid. Nature 469:424-427

Stremlau M, Perron M, Lee M, Li Y, Song B, Javanbakht H, DiazGriffero F, Anderson D, Sundquist W, Sodroski J (2006) Specific recognition and accelerated uncoating of retroviral capsids by the TRIM5alpha restriction factor. Proc Natl Acad Sci USA 103:55145519

Yang H, Ji X, Zhao G, Ning J, Zhao Q, Aiken C, Gronenborn AM, Zhang P, Xiong Y (2012) Structural insight into HIV-1 capsid recognition by rhesus TRIM5a. Proc Natl Acad Sci USA 109:18372-18377 